\title{
DEVELOPPEMENT LARVAIRE - REGIME ALIMENTAIRE, PRODUCTION D'ISOPERLA VIRIDINERVIS PICTET (PLECOPTERA, PERLODIDAE) DANS UN TORRENT FROID DE HAUTE MONTAGNE
}

\author{
par P. LAVANDIER ${ }^{1}$
}

Les populations larvaires d'Isoperla viridinervis ont été étudiées de 1971 à 1973 à 5 altitudes différentes entre la source (altitude : $2370 \mathrm{~m}$, Température maximale: $4,5^{\circ} \mathrm{C}$, enneigement : $6-8$ mois/an) et l'embouchure (altitude : $1850 \mathrm{~m}$, Température maximale : $13^{\circ} \mathrm{C}$, enmeigement : 45 mois/an) d'un torrent pyrénéen, l'Estaragne.

Les larves sont essentiellement carnivores et se nourrissent surtout de Baetidae et de Diamesinae. L'importance et la diversité des captures s'accroissent avec l'âge du prédateur.

Le développement larvaire dure 2 ans dans le cours inférieur et 3 ans dans le cours supérieur du torrent. Cet allongement réduit fortement le taux de survie des larves entre l'aval $(\sim 6 \%$ de survie moyenne) et l'amont $(<1 \%$ de survie moyenne). La mortalité a surtout lieu durant la période déneigée $-2 \%$ des larves disparaissent en moyenne chaque jour. Les effectifs sont relativement stables d'une année sur l'autre; les plus fortes variations s'observent en amont où le recrutement dépend en partie de la ponte de femelles venues d'aval.

La croissance s'effectue surtout en période déneigée. L'accroissement (en poids sec) des larves varie de 1 à $2,8 \%$ par jour. Il existe une relation hautement significative entre les taux de croissance instantanés et la température moyenne du milieu a'nsi qu'entre l'accroissement du poids individuel moyen des larves et le nombre de degrés-jours cumulés : 1967 degnés-jours sont nécessaires au développement larvaire d'Isoperla viridinervis dans le torrent.

La production est de $0,35 \mathrm{~g}$ de poids $\mathrm{sec} / \mathrm{m}^{2} / \mathrm{an}$ pour l'ensemble du cours. Les rapports Production/Biomasse moyenne d'une cohorte au cours de son développement varient de 4,8 à 8,4 . L'utilisation d'un rapport $\mathrm{P} / \overrightarrow{\mathrm{B}}=2,4$ permet d'obtenir une bonne estimation de la production annuelle à partir de la biomasse moyenne annuelle des populations, toutes cohortes confondues.

\section{Larval development, feeding and production of Isopenla viridinervis Pictet (Plecoptera, Perlodidae) in a cold river in the high mountains.}

The larval populations of Isoperla viridinervis have been studied from 1971 to 1973 af five different stations between the source (altitude : $2370 \mathrm{~m}$, maximum temperature $4.5^{\circ} \mathrm{C}$, snow cover for $6-8$ months/year) and the mouth (altitude: $1850 \mathrm{~m}$, maximum temperature: $13^{\circ} \mathrm{C}$, snow cover for $4-5$ months/year) of a fast-flowing Pyrenean river, Estaragne.

The larvae are essentially carnivores and feed chiefly on Baetidae and Diamesinae. The size and diversity of the prey increase with the age of the predator.

1. Laboratoire d'Hydrobiologie, ERA 7202 du CNRS, Université Paul-Sabatier, 118, route de Narbonne, 31062 Toulouse Cedex. 
Larval development takes two years in the lower course and three years in the upper course of the river. This extension markedly roduces the survival rate of the larvae between the lower $(\simeq 6 \%$ mean survival $)$ and upper $(<1 \%$ of mean survival) reaches. Mortality is especially high during the period without snow $-2 \%$ of the larvae disappearing on average each day. These effects are relatively stable from one year to another; the greatest variations occur in the upper reaches where recruitment partially depends upon the oviposition of females coming from the lower reaches.

Growth occurs chiefly during the period without snow. The increase (in dry weight) of the larvae varies from $1-2.8 \%$ per day. There is a highly significant relationship between the instantaneous growth rate and the mean temperature of the environment as well as between the mean individual weight of the larvae and the number of degree-days : 1967 degree days are necessary for the larval development of Isoperla viridinervis in the river.

Production is $0.35 \mathrm{~g}$ idry weight $/ \mathrm{m}^{2} /$ year for the whole of the river. The ratio of production to mean biomass for a cohort during its development varies from

4.8 to 8.4. Use of a ratio $P / \vec{B}=2.4$ provides a good estimate of annual production from the mean annual biomass of the populations, with all cohorts combined.

Une abondante littérature est consacrée au régime alimentaire et au cycle biologique des plécoptères carnivores de la famille des Perlodidae (cf. Hynes 1970, 1976 ; Berthélémy et Lahoud 1981); la dynamique des populations n'y est en revanche jamais totalement traitée sous son aspect quantitatif. Ce travail aborde la répartition, la nutrition, la croissance, la mortalité et la production d'une espèce endémique pyrénéenne, Isoperla viridinervis dans un torrent froid de haute montagne, l'Estaragne. Cette Isoperla, largement répandue dans les Pyrénées entre 1000 et $2400 \mathrm{~m}$ d'altitude (Berthélémy 1966) fait partie d'un groupe de plusieurs espèces morphologiquement très proches que l'on peut, comme dans le "complexe Isoperla grammatica " (Berthélémy 1979 b), différencier par leurs appels sexuels (Berthélémy comm. Verb.). Les individus étudiés dans le torrent d'Estaragne constituent un ensemble très homogène, certainement monospécifique.

\section{1. - MILIEU - MATERIEL - METHODE}

Le torrent d'Estaragne est situé dans les Pyrénées centrales. Il est issu d'un névé vers $2400 \mathrm{~m}$ d'altitude et 'se jette dans le lac d'Orédon à $1850 \mathrm{~m}$ d'altitude après un cours de $2,800 \mathrm{~km}$ environ. La sévérité du milieu s'accroît entre l'embouchure et la source, 'surtout en amont d'un ressaut glaciaire quil crée une importante rupture de pente entre 2000 et $1930 \mathrm{~m}$ d'altitude. Les caractéristiques du bassin-versant, l'évolution saisonnière du débit, de la température et de la composition chimique des eaux sont rapportées dans plusieurs publications consacrées à l'écologie de ce torrent (Lavandier 1974, Lavandier et Mur 1974, Lavandier 1981). Les cinq localités retenues dans ce travail correspondent aux stations dénommées A.B.R.C.D. dans ces publications. La situation et les principales caractéristiques de ces localités sont rappelées dans le tableau I.

Le torrent a été prospecté régulièrement de 1971 à 1973 durant la période d'accessibilité du vallon (juin-novembre). Quelques relevés ont été faits en hiver, période à laquelle peuvent subsister des parties déneigées (Décamps et Lavandier 
TABLEAU 1. - Caractéristiques principales des stations prospectées.

* Valeur tenant compte d'une température hivernale voisine de $0,5^{\circ} \mathrm{C}$ près de la source.

\begin{tabular}{|c|c|c|c|c|c|}
\hline & \multicolumn{5}{|c|}{ STATIONS } \\
\hline & A & B & $\mathrm{R}$ & c & D \\
\hline $\begin{array}{l}\text { Altitude (m) } \\
\text { Distance à la source }(\mathrm{m}) \\
\text { Largeur moyenne }(\mathrm{m})\end{array}$ & $\begin{array}{r}2370 \\
50 \\
1-2\end{array}$ & $\begin{array}{l}2190 \\
600 \\
2-3\end{array}$ & $\begin{array}{ll}2 & 150 \\
1 & 100 \\
& 3-5\end{array}$ & $\begin{array}{rr}1 & 920 \\
2 & 000 \\
3-5\end{array}$ & $\begin{array}{ll}1 & 850 \\
2 & 800 \\
& 4-6\end{array}$ \\
\hline $\begin{array}{l}\text { Durée d'enneigement (mois) } \\
\text { Température maximum }\left({ }^{\circ} \mathrm{C} \text { ) }\right. \\
\text { Nombre annuel de degrés-jours }\end{array}$ & $\begin{array}{l}6-8 \\
4,5 \\
588^{*}\end{array}$ & $\begin{array}{r}5-8 \\
7 \\
550\end{array}$ & $\begin{array}{l}5-7 \\
8,5 \\
650\end{array}$ & $\begin{array}{r}4-6 \\
11 \\
871\end{array}$ & $\begin{array}{r}4-5 \\
13 \\
1012\end{array}$ \\
\hline Conductivitë $20^{\circ} \mathrm{C}$ (umhos) & 114 & 108 & 93 & 87 & 80 \\
\hline
\end{tabular}

1970). Les larves étaient recueillies à l'aide ide filet de Surber de vide ide maille de 0,1 et $0,15 \mathrm{~mm}$.

A la station $\mathrm{R}$, dix à quinze relevés couvrant 1 à $1,5 \mathrm{~m}^{2}$ ont été réalisés toutes les 3 semaines en 1971, tous les mois en 1972 et 1973; aux autres stations, 4 à 6 relevés (couvrant environ $0,5 \mathrm{~m}^{2}$ ) ont été faits chaque mois. La faune était fixée au formol sur le terrain, triée au laboratoire puis conservée en alcool. Les adultes étaient capturés dans des pièges à émergence ou par chasse hebdamadaire.

\section{2. - REPARTITION}

Isoperla viridinervis peuple le torrent de la source à l'embouchure (tableau II). Son abondance est maximum entre 2100 et 2200 d'altitude où elle est en moyenne au cours de l'année de 440 individus $/ \mathrm{m}^{2}$. Elle diminue vers l'aval (50 individus $/ \mathrm{m}^{2}$ à l'embouchure) et vers l'amont (une centaine d'individus $/ \mathrm{m}^{2}$ près de la source) où l'espèce est supplantée par un autre Perlodidae Arcynopteryx compacta.

Tableau II. - Abondance moyenne en période déneigée, production annuelle et biomasses d'Isoperla viridinervis dans le torrent d'Estaragne.

\begin{tabular}{|c|c|c|c|c|c|}
\hline & \multicolumn{5}{|c|}{ STATIONS } \\
\hline & A & B & $\mathrm{R}$ & C & D \\
\hline Effectifs (N/m2) & 105 & 477 & 412 & 75 & 51 \\
\hline Production (mg/m2) & 111 & 838 & 560 & 162 & 92 \\
\hline $\begin{array}{l}\text { Biomasse maximale en période } \\
\text { déneigēe }(\mathrm{mg} / \mathrm{m} 2)\end{array}$ & 96 & 573 & 327 & 117 & 61 \\
\hline $\begin{array}{l}\text { Biomasse moyenne en période } \\
\text { déneigée }(\mathrm{mg} / \mathrm{m} 2)\end{array}$ & 53 & 297 & 244 & 67 & 37 \\
\hline
\end{tabular}


Isoperla viridinervis colonise tout le lit quels que soient le substrat et les conditions de courant; les larves âgées, toutefois, vivent préférentiellement parmi les gros blocs rocheux situés en courant vif.

\section{3. - REGIME ALIMENTAIRE}

Près de 300 larves provenant des localités d'amont ont été disséquées. Quatre périodes ont été considérées - hiver, fonte des neiges, septembre, novembre et à chacune d'elles la nutrition des cohortes d'âge diffénent qui coexistent dans le milieu a été analysée séparément. L'importance du contenu intestinal a été estimée par un indice de réplétion allant de 0 (intestin vide) à 5 (intestin plein). Les résultats font l'objet du tableau III, les différences entre stations sont précisées dans le texte.

La quantité de nourriture ingérée est peu abondante en hiver, augmente à la fonte des neiges, passe par un maximum au cours de l'été puis diminue en automne. La proportion d'individus capturés à jeun suit une évolution parallèle mais reste inférieure à $40 \%$ excepté chez les larves-nymphes qui ne se nourrissent plus avant l'émergence; elle est toujours plus forte chez les larvules que chez les larves de taille moyenne.

Les invertébrés constituent la plus grande part de l'alimentation d'Isoperla viridinervis; les algues, fréquemment ingérées en petites quantités, surtout par les plus jeunes larves, deviennent toutefois l'élément essentiel de l'alimentation en fin d'année : plus de $70 \%$ des estomacs en sont alors abondamment garnis, à l'exclusion souvent de toute proie animale. Les éléments minéraux fins sont fréquents chez les jeunes Isoperla; ils disparaissent peu à peu du contenu intestinal au cours du développement de l'espèce.

Le nombre moyen des proies par intestin s'accroît légèrement avec l'âge du prédateur (fig. 1 a). Les rares valeurs éloignées de la moyenne se rapportent toutes à d'abondantes captures de larves néonates.

Les Chironomidae (Orthocladiinae et Diamesinae) et les Baetidae constituent les $9 / 10$ des invertébrés capturés par $I$. viridinervis au cours de sa vie larvaire. Le reste de l'alimentation est constitué par Rhithrogena loyolaea, Leuctra spp, auxquelles s'ajoutent dans le bas du vallon quelques Simuliidae et Thaumaleidae. Les formes carnivores (Rhyacophila spp., Arcynopteryx compacta) ou protégées par un fourreau (Drusinae, Tanytarsini) sont respectées. Le cannibalisme est peu développé : 2 larvules d'Isoperla seulement auraient été ingérées par leurs congénères. Les Oligochètes paraissent également dédaignés bien que trois des larvules d'Isoperla disséquées renfermaient des soies de Trichodrilus.

L'abondance relative des espèces capturées diffère selon la composition des populations benthiques. Au cirque supérieur la prédation 
Tableau III. - Régime alimentaire d'Isoperla viridinervis.

Largeur capsule céphalique (en $1 / 10 \mathrm{~mm}$ )

Effectif observê Degré moyen de réplétion

Nombre d'intestins

- vides

- avec minéraux

- avec végétaux

- avec animaux

Effectif des proies ingērēes

- Simuliidae

- Diamesinae

- Orthocladicinae

- Chironomidae (indéterminês)

- Baetis alpinus

- Rhithrogena Toyolaea

- Isoperla viridinervis

- Leuctra rauscheri

- Leuctra SD.

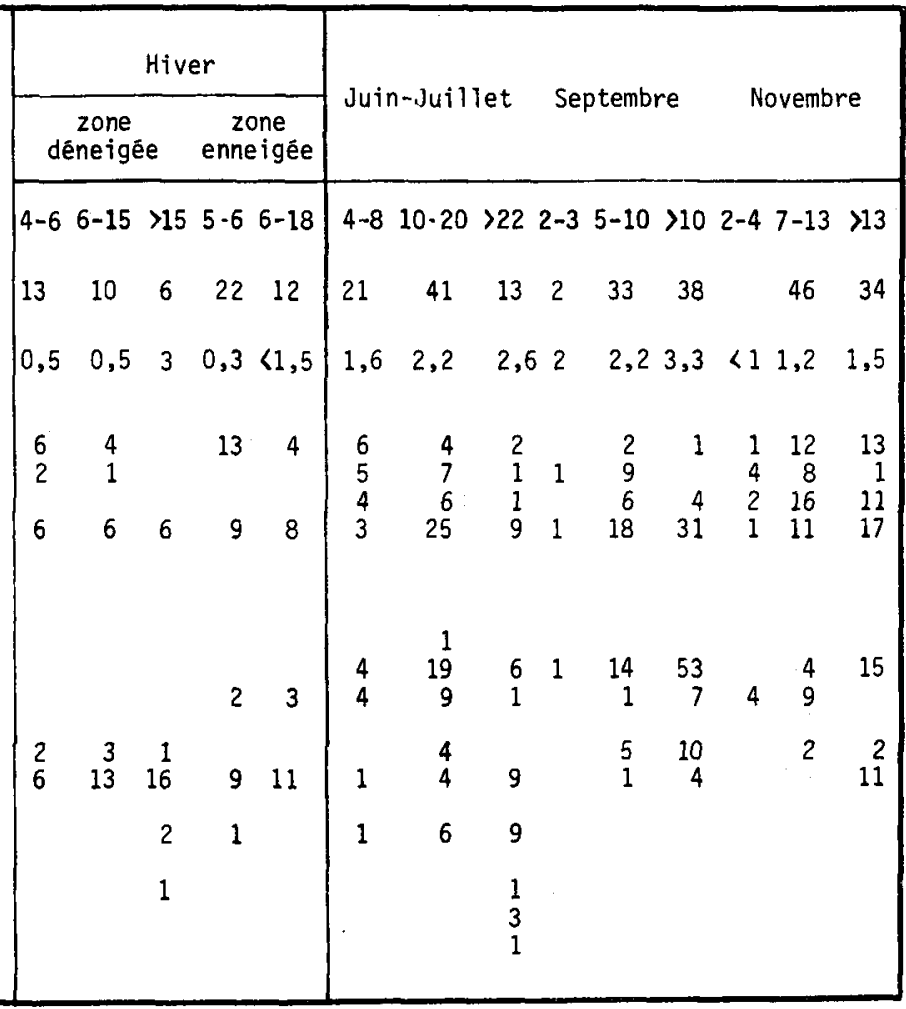

affecte essentiellement les Chironomidae qui sont les invertébrés les plus abondants. Ailleurs, les Chironomidae (qui dominent en été) et les Baetidae (qui dominent en hiver) sont tour à tour les plus consommés. Les Chironomidae, qui sont toujours proportionnellement plus nombreux parmi la faune ingérée qu'au sein du benthos, constituent néanmoins la proie préférentielle du plécoptère : les captures de $R h i$ throgena loyolaea, limitées à la période de crue nivale, traduisent vraisemblablement la superposition partielle et momentanée des habitats de la proie et du prédateur à cette époque, caractérisée par une migration larvaire importante.

La taille des invertébrés consommés varie comme la taille du prédateur (fig. 1 bc). Cette relation est plus étroite pour les Baetidae que pour les Chironomidae dont seules les larves les plus petites et les plus grosses échappent à une partie des populations d'Isoperla. Les larves ingérées reflètent la structure générale des populations auxquelles elles appartiennent (fig. $1 \mathrm{c}$ ). 


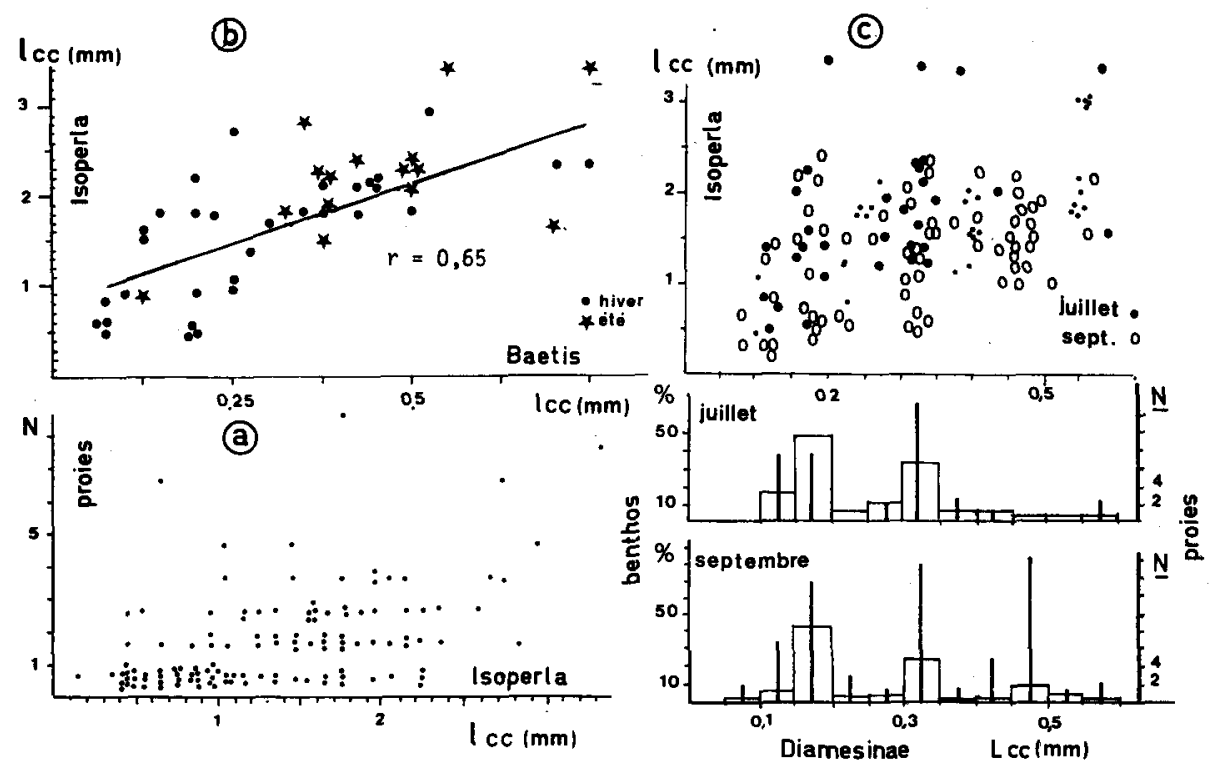

Fig. 1. - Caractéristiques de la nutrition d'Isoperla viridinervis.

a) relation taille du prédateur - nombre de captures.

b) relation taille du prédateur - taille des larves de Baetis ingérées.

c) prédation des Diamesinae

- en haut : relation taille du prédateur - taille des larves ingérées.

- en bas : relation taille et abondance des larves ingérées (traits verticaux) structure ides populations benthiques (histogramme) à 2 périodes de l'année.

\section{4. - DEVELOPPEMENT LARVAIRE}

Le développement larvaire est déduit de l'évolution de la structure des popu lations au cours de l'année. La largeur des capsules oéphaliques yeux compris ( $\mathrm{cc}$ en $\mathrm{mm}$ ) sert de critère de taille. On peut en déduire la longueur moyenne des larves $(\mathrm{L}$ en $\mathrm{mm}$ ) par la relation $\mathrm{L}(\mathrm{mm})=4,94 \mathrm{l} \mathrm{cc}(\mathrm{mm})-0,5$.

Le développement larvaire dure 2 ans au-dessous de $1900 \mathrm{~m}$ d'altitude et 2 ou 3 cohortes sont présentes simultanément dans le milieu (fig. 2 b). Les larvules éclosent de juillet à septembre. Le développement larvaire est immédiat; il fléchit en hiver puis reprend vigoureu. sement dès la fonte des neiges. Au début du deuxième hiver la taille moyenne des capsules céphaliques est de $2 \mathrm{~mm}$. Le cycle s'achève l'année suivante, les adultes volent de juin à septembre.

Dans la partie haute du vallon, au-dessus de $2100 \mathrm{~m}$ d'altitude, le développement larvaire dure trois ans et 3 ou 4 cohortes coexistent en permanence (fig. 2 a). Les éclosions se produisent surtout d'août à octobre mais les larvules sont encore abondantes en fin d'année près 

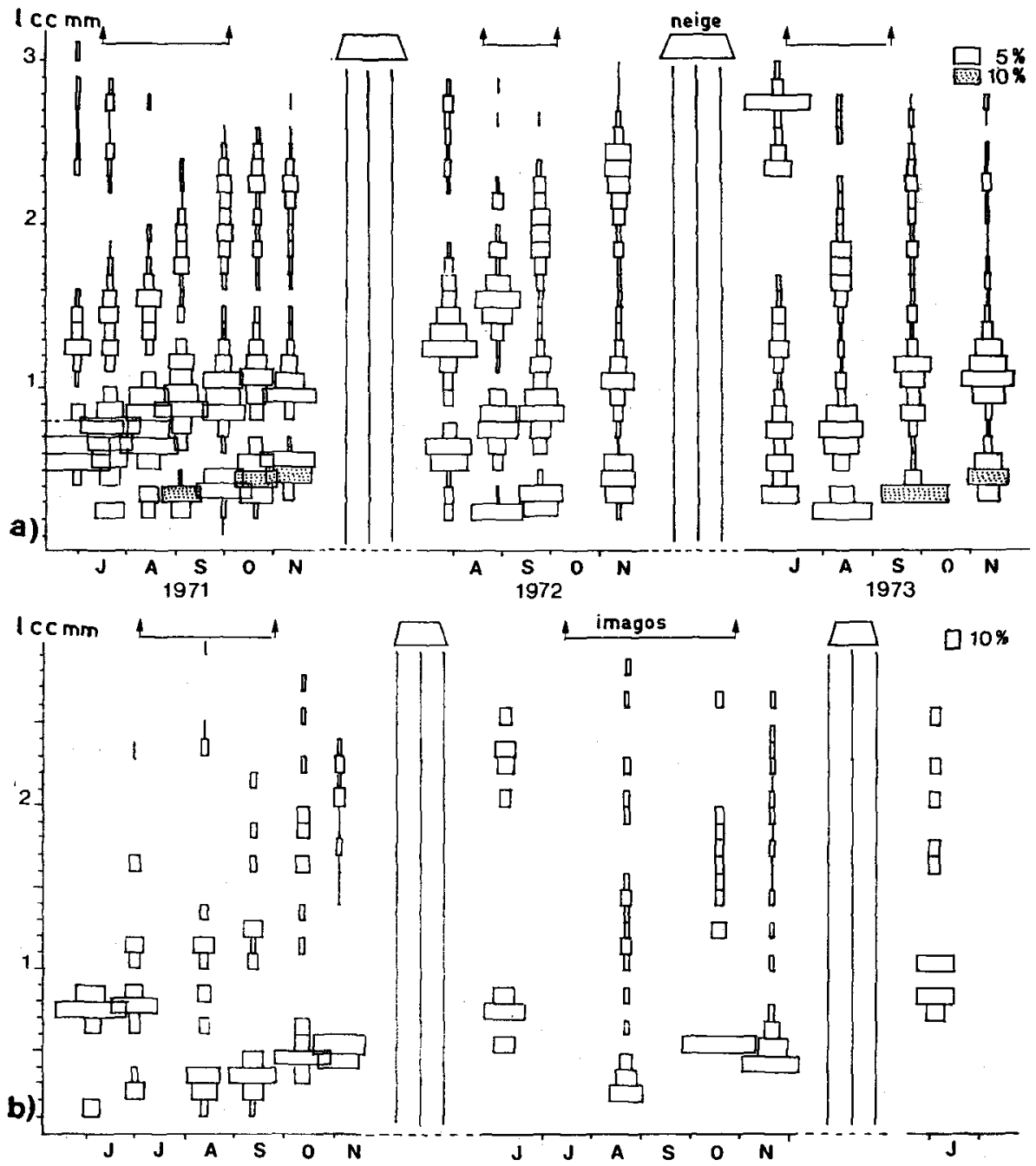

FIG. 2. - Développement larvaire d'Isoperla viridinervis.

a) dans le cours supérieur du torrent d'Estaragne (au-dessus de $2100 \mathrm{~m}$ ).

b) dans le cours inférieur (au-dessous de $1950 \mathrm{~m}$ ).

de la source. Le développement, négligeable sous la neige, s'accélère au cours de l'été mais au début du deuxième hiver, la largeur moyenne des capsules céphaliques est encore inférieure au mm. Au ralentissement hivernal de la croissance, fait suite une nouvelle reprise estivale du développement mais l'espèce doit subir un troisième hiver avant de s'envoler après la crue nivale. Le début des émergences se retarde d'août à octobre entre $2150 \mathrm{~m}$ (station $\mathrm{R}$ ) et $2370 \mathrm{~m}$ d'altitude 
(station $\mathrm{A}$ ) où quelques individus n'achèvent vraisemblablement pas leur développement avant le quatrième hiver.

Les proportions d'adultes mâles et femelles capturés par chasse s'inversent entre l'aval et l'amont du torrent : 76 o pour 52. au niveau du lac, 34 of pour $25 \&$ vers 2100 m d'altitude, 9 ô pour $24 \%$ près de la source. Cette inversion traduit une nette migration des femelles vers l'amont, migration que confirment les captures de femelles gravides faites chaque année en juillet aux stations supérieures, donc avant le début des émergences à leur altitude.

\section{5. - EVOLUTION NUMERIQUE}

Par analogie avec les principales espèces de l'Estaragne (Lavandier 1979 a) la réduction des effectifs d'Isoperla viridinervis a été assimilée à une fonction exponentielle décroissante en fonction du temps : $\mathbf{N t}=\mathrm{No}^{-\mathrm{zt}}$ avec $\mathrm{Nt}$.et $\mathbf{N o}=$ effectifs aux temps $t$ et to, $t$ en jours, $z=$ taux instantané de diminution des nombres.

Après la. période d'éclosion des larvules caractérisée par un brusque accroissement des effectifs, l'évolution numérique des cohortes dessine chaque année une courbe en cloche dont le mode se situe en septembre ou en octobre selon les années et l'altitude des localités (fig. 3). ' L'augmentation apparente des effectifs qui a lieu pendant la. décrue traduit la recolonisation progressive de l'ensemble du lit par la faune à partir des habitats refuges qu'elle occupait lors des hautes eaux; parallèlement la distribution de la faune s'homogénéise sur le fond. - La diminution des effectifs qui s'observe à l'étiage résulte de la mortalité naturelle ( + dérive) des larves.

Si on admet une décroissance exponentielle des effectifs, $2 \%$ des larves disparaissent en moyenne chaque jour durant la saison déneigée, en dehors des périodes d'éclosion et d'émergence (tableau IV). La diminution des nombres est beaucoup moins rapide durant la phase hivernale et la crue de fonte des neiges : $0,25 \%$ des effectifs disparaissent en moyenne chaque jour dans le réseau hydrographique entre les derniers prélèvements de l'année (mi-novembre) et les relevés les plus riches de l'été suivant. Compte tenu de la durée du développement, la survie peut-être estimée à $0,7 \%$ dans la partie haute du torrent (station A $0,5 \%$, St. B $0,16 \%$, St. R $1,5 \%$ ) et à $8 \%$ dans la partie basse (St. C 9,6\%, St. D 6,3\%).

Les effectifs des diverses cohortes coexistantes sont relativement stables près du lac où ils sont chaque année du même ordre. En amont, le nombre de jeunes larves présente en revanche des différences significatives d'une année sur l'autre $(\mathrm{P}=0,05)$; les plus importantes s'observent près de la source où le recrutement est fonction de la ponte de $q$ venues d'aval. Les variations initiales s'amenuisent 
40
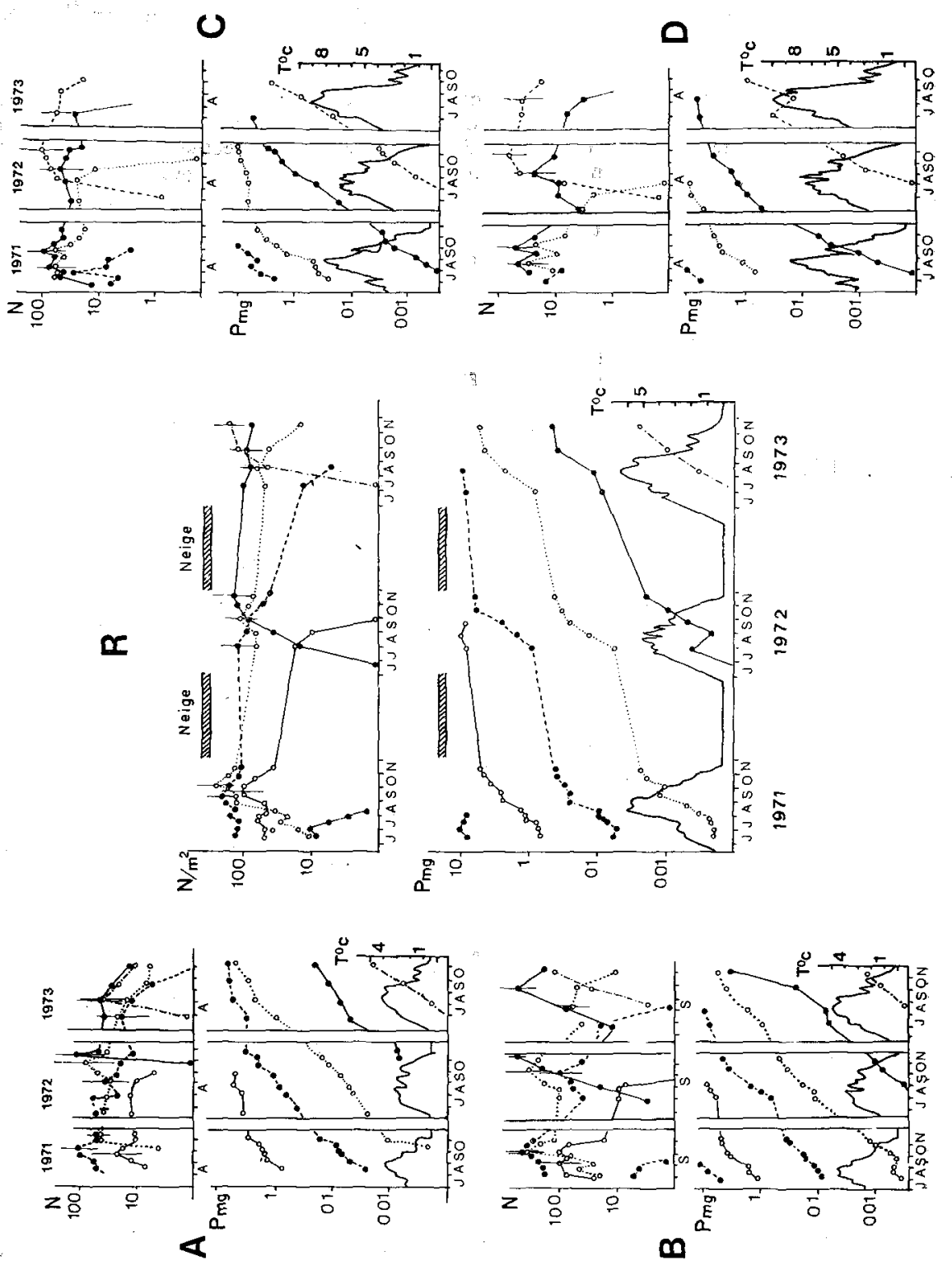

Fig. 3. - Evolution numérique et croissance du poids individuel moyen des larves de chaque cohorte aux 5 stations étudiées. L'évolution saisonnière de la température est indiquée sur les graphiques relatifs à la croissance pondérale. Les limites de confiance (à $95 \%$ ) des estimations sont indiquées pour les effectifs maxima observés chaque année. 
au cours du développement et dans la troisième anmée de vie larvaire, les effectifs ne diffèrent pas significativement d'une année sur l'autre.

TableaU IV. - Taux journaliers de croissance du poids individuel moyen des larves (K) et taux journaliers de diminution des nombres ( $\mathrm{Z}$ ) établis pour chaque cohorte à partir de la fig. 3. - Les valeurs affectées d'un astérique concernent des périodes non exemptes d'éclosions ou d'émergences.

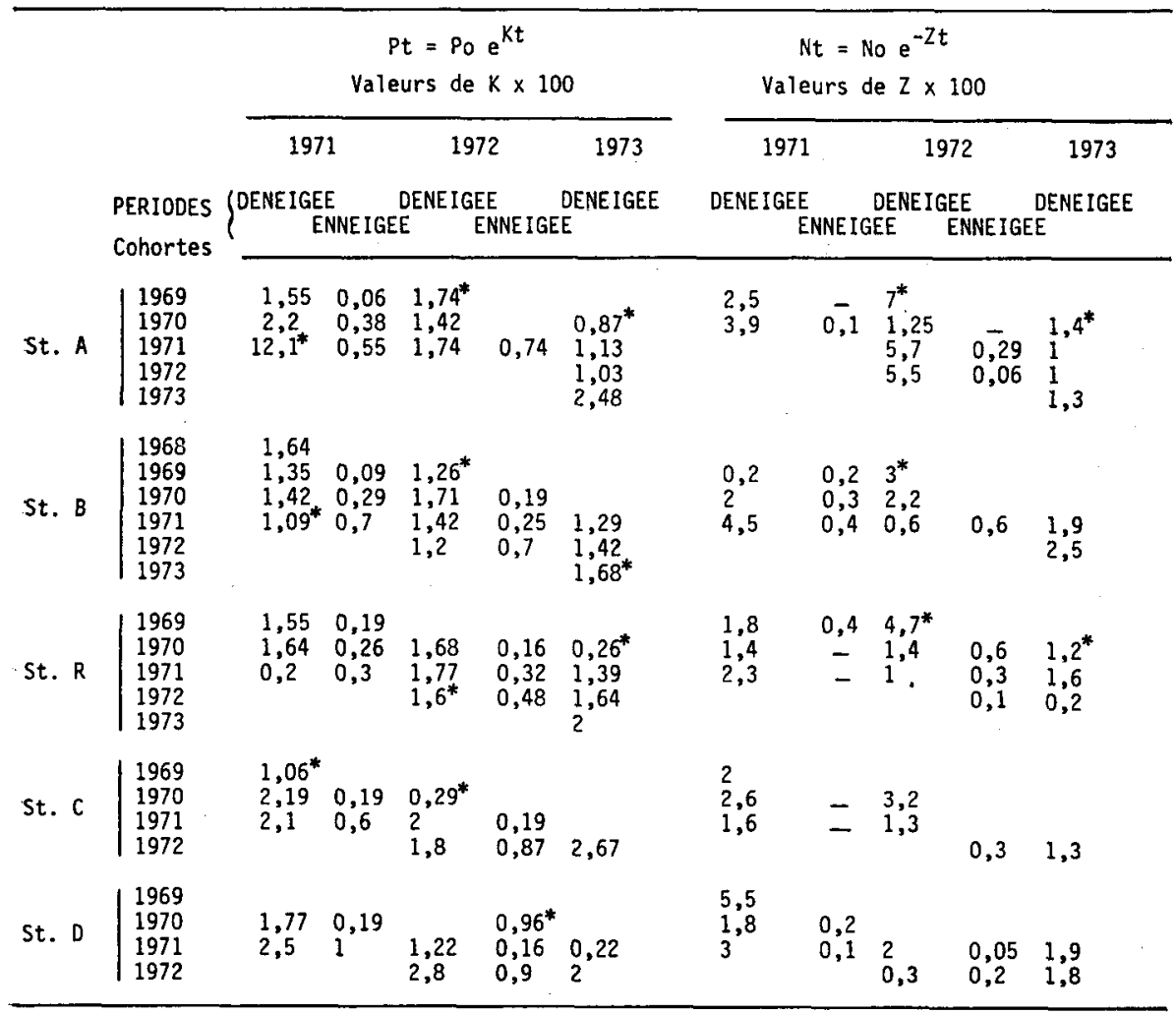

\section{6. - CROISSANCE PONDERALE}

La croissance est estimée d'après l'évolution pondérale des larves en fonction du temps. Dans les divers relevés le poids individuel moyen des larves de chaque cohorte est calculé à partir du poids des effectifs de chaque classe de taille déduit de la relation: $\log P s=3,3 \log I-0,99 \pm 0,34$ (limites de confiance à $95 \%), N=10, r=0,99, \mathrm{Ps}=$ poids sec des larves en mg après dessèchement à l'étuve à $80^{\circ} \mathrm{C}$ pendant $24 \mathrm{~h}, 1=$ largeur des capsules céphaliques en $\mathrm{mm}$.

L'augmentation du poids moyen des larves dans chaque cohorte a été assimilée pour les phases enneigóes et déneigées successives à des portions de courbes exponentielles en fonction du temps $\mathrm{Pt}=\mathrm{Po}$ eKt avec $\mathrm{Pt}$ et $\mathrm{Po}=$ poids moyen des larves aux temps $t$ et to, $t$ en jour, $K=$ taux de croissance instantané mg $\mathrm{mg}^{-1}$ jour ${ }^{-1}$. 
La croissance larvaire est faible sous la neige, beaucoup plus forte durant la phase déneigée (fig. 3). Elle est peu perturbée par les fluctuations hydriques et se ralentit légèrement en fin d'année. $K$ est en moyenne de 0,003 en hiver et varie de 0,01 à 0,028 en phase déneigée (tableau IV). En dehors des périodes d'éclosion et d'émergence il existe une relation hautement significative entre le taux de croissance instantané des larves $\mathrm{K}$ et la température moyenne du milieu $\mathrm{K}=0,0035 \pm 0,00068 \mathrm{~T}+0,00222 \pm 0,0088, \mathrm{r}=0,81$ (fig. 4); la relation est légèrement plus étroite lorsque la température est exprimée en Jogarithmes $\mathrm{K}=0,0088 \pm 0,0014 \log \mathrm{T}+0,0052 \pm 0,0078 \mathrm{r}=0,85$ ce qui souligne l'importance des fluctuations thermiques lorsque les températures sont basses.
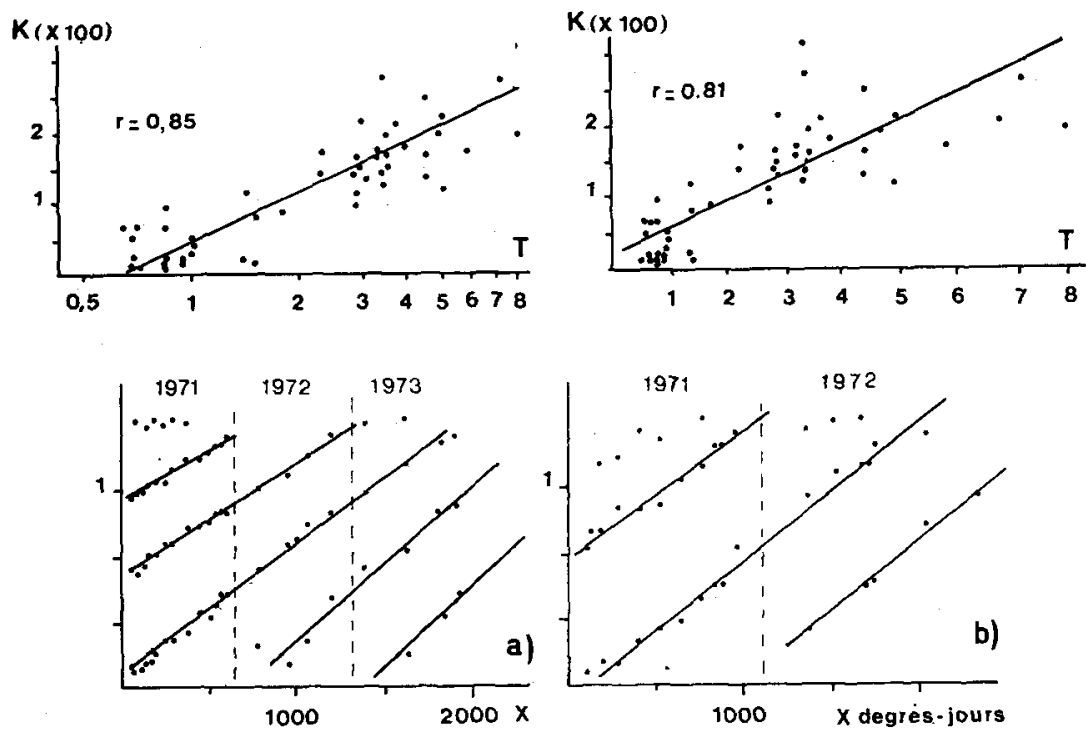

Fig. 4. - - en haut : Relation entre le taux de croissance instantané des larves

(K) et la température moyenne du milieu ( $\mathrm{T}$ ou Log T).

- en bas: Evolution du poids individuel moyen des larves en fonction du nombre de degrés-jours cumulés

a) dans le cours supérieur (station $R$ ).

b) dans le cours inférieur (station $\mathbf{C}$ ).

La relation entre la croissance et la température a été précisée en confrontant l'évolution du poids individuel moyen des larves au nombre de degré-jours cumulés (fig. 4), c'est-à-dire au temps pondéré par la température (cf. Wimberg 1971, Markarian 1980). Calculée en dehors des phases d'éclosion et d'émergence, cette relation est hautement significative à chaque station et pour chaque cohorte; elle est de la forme $\log P x=A x+\log$ Po avec $x=$ nombre de degrés-jours, $P x=$ 
poids des larves pour une somme thermique $x$. Le taux d'accroissement moyen A mg mg ${ }^{-1}{ }^{\circ} \mathrm{j}^{-1}$ des diverses populations varie de 0,00469 près de la source à 0,00425 à l'embouchure. Calculé sur l'ensemble des cohortes il est en moyenne de 0,00445 $\pm 0,00044$. Si l'on admet une température-seuil proche de $0{ }^{\circ} \mathrm{C}$, la somme thermique nécessaire au développement des larves varie de 1860 degrés-jours près de la source à 2050 près du lac pour une valeur moyenne de 1967 (L.C. $95 \% 1790-2183$ ) sur l'ensemble du torrent.

\section{7. - PRODUCTION LARVAIRE}

La production est calculée d'après la méthode graphique d'Allen (1951). Son application aux invertébrés de l'Estaragne a été discutée par ailleurs (Lavandier 1975, 1979 a et $b, 1981$ ) et en particulier: 1) une courbe unique de croissancesurvie est tracée à partir des résultats obtenus au cours des 3 années id'étude. 2) il n'est pas tenu compte des relevés relatifs aux périodes de crues - au cours desquelles une partie des populations migre à l'abri du courant (Lavandier 1979 a) ni des captures des larves néonates et matures qui, compte tenu de l'étalement des éclosions et des émergences ne peuvent être représentatives des effectifs réels en début et en fin de développement. La production est restimée en prolongeant la courbe de croissance-survie jusqu'aux points d'abscisse $P o=$ $0,0017 \mathrm{mg}$ poids des plus petites larves récoltées et $\mathrm{Pl}=10,8 \mathrm{mg}$ poids moyen des larves en fin de développement ; cette extrapolation est facilitée par le calcul de l'équation des portions initiales et terminales des courbes de croissance-survie correspondant d'une part aux larves jeunes et id'autre part aux larves âgées, en considérant que la mortalité et la croissance suivent toutes deux des courbes exponentielles (Ness et Dugdale 1959):

La production la plus forte s'observe entre 2100 et $2200 \mathrm{~m}$ (Tableau II) où les populations sont les plus abondantes. Les stations d'aval sont moins riches et moins productives. Toutefois, la production n'y est en moyenne que cinq fois plus faible alors que les effectifs y sont sept fois moindres; elle est en outre pilus forte que près de la source où l'espèce est pourtant mieux représentée : en aval, la mortalité plus faible et le cycle plus court compensent totalement le déficit initial des effectifs et, en fin de développement, les larves âgées qui déterminent l'essentiel de la production sont plus nombreuses qu'à la source. L.'estimation des effectifs néonates et matures à partir des courbes de croissance-survie conduit à des taux moyens de survie de 0,6\% en amont (Station A $1,2 \%$, St. B $0,2 \%$, St R $0,4 \%$ ) et de $3,75 \%$ en ava! (St. C 6,5\%, St. D 1\%). Les deux tiers de la production sont assurés par la cohorte qui doit émerger l'année suivante (cohorte $1^{+}$ au-dessous de $2000 \mathrm{~m}$, cohorte $2^{+}$au-dessus) ; plus de $70 \%$ du poids sec sont produits dans la deuxième partie du cycle biologique. Le rapport entre la production et la biomasse moyenne en période déneigée est en moyenne de 2,40 . 


\section{8. - DISCUSSION}

Le régime alimentaire d'Isoperla viridinervis rappelle celui d'Isoperla moselyi et d'I. acicularis (Berthélémy et Lahoud 1981), deux espèces présentes mais rares dans le bas du torrent; plus généralement il correspond à celui des Isoperla des groupes grammatica et rivulorum (cf. Berthélémy et Lahoud, op. cit.). La part du matériel végétal dans l'alimentation diminue au cours de la vie larvaire. Chironomidae (Orthocladiinae et Diamesinae) et Baetidae sont les invertébrés les plus consommés mais l'abondance relative des espèces ingérées par rapport à la composition de la faune benthique (Hunt et Jones 1972, Siegfried et Knight 1976) indique que ce sont surtout les Chironomidae qui sont recherchés de façon préférentielle; ils sont en fait vulnérables à la grande majorité de la population d' $I$. viridinervis durant tout leur développement alors que la taille des Baetidae dépend davantage de celle du prédateur; cette différence entre captures d'éphémeroptères et de diptères a été plusieurs fois observée par ailleurs (Sheldon 1969, Winterbourn 1974, Mouthon et Verneaux 1978, Berthélémy et Lahoud 1981). Les caractéristiques de l'alimentation du prédateur reflètent en fait ses besoins nutritifs, ses capacités de prénension - liées à la taille et au comportement de chasse ou de fuite des antagonistes (Hynes 1941, Peckarsky 1980, Malmquist et Sjöström 1980) - et surtout la disponibilité de la faune à son égard (Tachet 1965, Siegfried et Knight 1976, Lavandier 1979 a, Gray et Ward 1979).

Ainsi, il est vraisemblable qu'I. viridinervis rampe sur le fond, happant au passage les larves qu'il rencontre. Jeune, il s'immisce entre les graviers où il capture des larvules et des oligochètes, ingérant alors de fins éléments minéraux; plus âgé son rayon d'action s'élargit, ses besoins nutritifs augmentent et sa nutrition se diversifie : captures plus. nombreuses et de tailles variées, algues; les minéraux se raréfient. En fin de vie larvaire, les interstices du sustrat deviennent inac. cessibles au prédateur: les éléments minéraux et les larvules disparaissent de son contenu intestinal, au profit de larves plus âgées fréquemment capturées au sein des hydrures en été (Baetis, Chironomidae) ou dans les habitats refuges à la fonte des neiges (Rhithrogena).

Le développement larvaire est plus long que celui de la plupart des Perlodidae (of. Hynes 1970, Harper 1973, Hynes 1976, Krueger et Cook 1981); seul Arcynopteryx compacta, d'ailleurs présent dans la partie amont du torrent, se développe sur une aussi longue durée (Lavandier 1979 b, Lillehamer 1979). Il se rapproche en revanche du développement des Eustheniidae, famille de plécoptères australiens (Hynes et Hynes 1975) avec lesquels il partage la particularité, rarement constatée chez les plécoptères de l'hémisphère nord, d'avoir un 
cycle variable selon les conditions de milieu (Brittain 1978, Lillehamer 1975. Hynes 1976, Hildrew et al. 1980).

En outre, la présence quasi permanente de larvules en période déneigée - parfois même avant le début des émergences - semble indiquer que le développement embryonnaire peut être de longue durée et que les éclosions sont étalées. Ceci, fréquent chez les Perlodidae (Khoo 1968 - Schwarz 1970 - Harper 1973 - Berthélémy 1979 a) a d'ailleurs été observé sur plusieurs pontes d'Isoperla viridinervis mises en incubation au laboratoire (Berthélemy comm. verb.). Ainsi, compte tenu du développement des œufs, le cycle biologique dure vraisemblablement 3 ou 4 ans selon l'altitude pour une partie des populations.

La. longueur du développement larvaire a pour conséquence le maintien clans le milieu de larves de taille variée qui utilisent au maximum les ressources disponibles et exercent une pression permanente sur la faunc benthique (Peckarsky et Dodson 1980).

L'allongement du cycle biologique signalé pour plusieurs espèces de trichoptères et d'éphéméroptères entre l'aval et l'amont du torrent d'Estaragne (Décamps 1967, Lavandier 1979 a, 1981) résulte ici dovantage de la réduction de la période déneigée que de la diminution de la vitesse de croissance lorsque l'altitude augmente. Cet allongement provoque une baisse importante du taux de survie qui, selon la. méthode d'estimation, est en moyenne 6 ou 11 fois plus faible dans 1a partie haute que dans la partie basse du cours. Bien que le nom. bre de pontes déposées dans le torrent et le pourcentage de réussite des éclosions soient indéterminés, la survie larvaire, que les diverses estimations chiffrent à environ $3 \%$ pour l'ensemble du réseau hydrographique, est parfaitement compatible avec le pouvoir reproducteur de l'espèce tel qu'on peut l'envisager grossièrement à partir du nombrc d'œufs comptés dans l'abdomen de femelles gravides $=484$ à 792 œufs par femelle - moyenne 598 cufs pour 14 dénombrements - La proportion de larves néonates d'Isoperla viridinervis qui parvient au terme de leur développement est du même ordre que chez Rhithrogenc: loyoiaea mais cing fois moindre que chez Arcynopteryx compacta pou: des durées de développement semblables dans le torrent (Lavandier 1979 b, 1981).

Les adultes femelles d'Isoperla viridinervis migrent vers l'amont. Ce déplacement qui permet de compenser les pertes par dérive des larves (Muller 1954) a rarement été signalé chez les plécoptères (Schwarz 1970, Muller 1973, 1982, Madsen et al. 1973, Zwick 1980. Il est ici en partie responsable des fluctuations annuelles du recrutement dans la partie amont alors que les effectifs à l'émergence sont chaque annéc du même ordre. Cette relative stabilité numérique qui se retrouve à chaque niveau dès la deuxième partie du cycle biologique 
reflète certainement, dans un cours d'eau soumis à de profondes perturbations saisonnières, les "capacités de charge maximum » lorsque les conditions sont les plus difficiles.

La croissance pondérale est en moyenne de $0,3 \% \mathrm{~J}^{-1}$ en hiver et de $1,5 \% \mathrm{~J}^{-1}$ en période déneigée. Cette dernière valeur est supérieure aux estimations faites chez un Perlidae américain (Heiman et Knight 1975); elle est en revanche légèrement plus faible que pour Arcynopteryx compacto (Lavandier $1979 \mathrm{~b}$ ) et que pour Rhithrogena loyolaea (Lavandier 1981) dont les modalités de développement son voisines de celles d'isoperla viridinervis dans le torrent. La relation qui existe entre le taux de croissance instantané $K$ et la température moyenne du milieu ( $\mathrm{T}$ ou $\log \mathrm{T}$ ) indique que les variations de $\mathrm{K}$ sont surtout fonction de la température. Le fait que cette relation soit légèrement plus étroite lorsque la température est exprimée sous forme logarithmique montre, en outre, que les variations de température ont une incidence d'autant plus importante sur la croissance que les températures sont faibles. Ceci se retrouve au niveau des sommes thermiques nécessaires au développement qui sont légèrement moindres à la source qu'à l'embouchure.

Si la température paraît constituer le principal facteur déterminant la. croissance, une part importante des variations de $\mathrm{K}$ en est indépendante. Les paramètres qui en sont responsables doivent être recherchés dans les fluctuations du niveau qui induisent en partie le comportement de la faune et dans les modifications de l'alimentation qui peuvent influer sur le développement (Anderson et Cummins 1979, Gray et Wand 1979, Peckarsky 1980, Sutcliffe et al. 1981).

La construction d'une courbe unique de aroissance-survie à chaque station conduit à l'évaluation de la production moyenne d'une cohorte à partir des productions partielles des cohortes coexistantes. Elle correspond également à la production moyenne annuelle de la population. Durant la période d'étude, $0,35 \mathrm{~g}$ de poids $\mathrm{sec} / \mathrm{m}^{2}$ ont été produits en moyenne chaque année. Les rapports $\mathrm{P} /$ Biomasse moyenne d'une cohorte au cours de son développement varient de 4,8 à 8,4 ; ces rapports sont supérieurs aux estimations théoriques de Waters (1969) et aux valeurs de 4 et 5 retenues par Lapchin et Neveu (1980) et Waters (1979) pour estimer la production d'une cohorte. Les rapports $\mathbf{P} / \mathbf{B}$ qui expriment la production annuelle (équivalente à. la production d'une cohorte) par rapport à la biomasse moyenne (toutes cohortes confondues), lorsque le milieu est accessible (biomasse voisine de la biomasse moyenne annuelle) varient de 2 à 2,8 et la valeur moyenne de 2,4 permet d'obtenir une bonne estimation de la production à partir de la biomasse échantillonnée. 


\section{TRAVAUX CITES}

Allen (K. R.). 1951. - The Horokiwi stream - A study of a trout population. Fish. Bull. N. Z., 10 : 1-231.

Anderson (N. H.) and Cummins (K. W.). 1979. - The influences of diet on the life histories of aquatic insects. Journal of the Fisheries Research Board of Canada, 36 (3) : 335-342.

BERTHÉLÉMY (C.). 1966. - Recherches écologiques et biogéographiques sur les Plécoptères et Coléoptères d'eau courante (Hydraena et Elminthidae) des Pyrénées. Annls Limnol., 2 (2) : 227-458.

BERTHÉLÉMY (C.). 1979 a. - Accouplement, période d'incubation et premiers stades larvaires de Brachyptera braueri et de Perlodes microcephalus (Plecoptera). Annls Limnol., 15 (3) : 317-335.

BerthÉLÉmy (C.). 1979 b. - Mating calls and taxonomy in Pyrenean Isoperla. Gewäss-Abwäss., 64 : 71-72.

Berthélémy (C.) et LAHoud (M.). 1981. - Régimes alimentaires et pièces buccales de quelques Perlodidae et Perlidae des Pyrénées (Plecoptera). Annls Limnol., 17 (1) : 1-24.

BrITtaIN (Y. E.). 1978. - Semivoltinism in mountain populations of Nemurella picteti (Plecoptera). Oikos, $30: 1-6$.

DÉcamps (H.). 1967. - Ecologie des Trichoptères de la vallée d'Aure (HautesPyrénées). Annls Limnol,, 3 : 399-577.

Décamps (H.) et LAVANDiER (P.). 1970. - Proceedings of the I B P UNESCO Symposium on Productivity Problems of Freshwaters. Kazimierz Dolny, Poland., 875-881.

GRAY (L. J.) et WARD (J. V.). 1979. - Food habits of stream benthos at sites of differing food avoilability. Am. Midl. Nat., 102 (1) : 157-167.

HARPER (P. P.). 1973. - Emergence, reproduction and growth of Setipalpian Plecoptera in southern ontario. Oikos 24 (1) : 94-107.

Heiman (D. R.) et KNIGHT (A.W.). 1975. - The influence of temperature on the bioenergetics of the carnivorous stonefly nymph, Acroneuria californica Banks (Plecoptera : Perlidae). Ecology, 56: 105-116.

Hildrew (A. G.), Townsend (C. R.) et Henderson (J.). 1980. - Interactions between larval size, microdistribution and substrate in the stoneflies of an iron-rich stream. Oikos $35:$ 387-396.

Hunt (P. C.) et Jones (J.W.). 1972. - The food of brown trout in Llyn Alaw, Anglesy, North wales. J. Fish. Biol., $4: 182-205$.

HYNES (H. B. N.). 1941. - The taxonomy and ecology of the nymphs of British Plecoptera with notes on the adults and eggs. Trans. r. ent. London., 91 : $455-55 i$.

HyNEs (H. B. N.). 1970. - The ecology of Running Waters. Liverpool university Press. Liverpool, $555 \mathrm{p}$.

Hynes (H. B. N.). 1976. - Biology of Plecoptera. Annu, Rev. Entomol., 21 : 135153.

Hynes (H. B. N.) et Hynes (M.). 1975. - The Life Histories of Many of the Stoneflies (Plecoptera) of South-eastern Mainland Australia. Aust. J. mar. Freshwai., Res., $26:$ 113-53.

Kroo (S. G.). 1968. - Experimental studies on diapause in Stoneflies. Proc. $r$. eni. Soc. London (A)., 43 : 40-56.

KRUEGER (C.C.) et CoOK (F. F.). 1981. - Life cycles, drift, and standing stocks of some stoneflies (insecta: Plecoptera) from streams in Minnesota, U.S.A. Hydrobiologia, 83 (1) : 85-92.

LAPCHIN (L.) et NEveU (A.). 1980. - The production of benthic invertebrates : com- 
parison of different methods II. Application to the benthos of the Nivelle river (Pyrénées-Atlantiques, France). Acta Oecologia Oecol. Gener., 1 : 359. 372.

Lavandier (P.). 1974. - Ecologie d'un torrent pyrénéen de haute montagne; I. Caractéristiques physiques. Annls Limnol., 10 (2) : 173-219.

LaVANDIER (P.). 1975. - Cycle biologique et production de Capnioneura brachyptera D. (Plécoptères) dans un ruisseau d'altitude des Pyrénées centrales. Annis Limnol., 11 (2) : 145-156.

LAVANDIER (P.). 1979 a. - Ecologie d'un torrent pyrénéen de haute montagne: l'Estaragne. Thèse sciences Université Paul Sabatier Toulouse, $532 \mathrm{p}$.

Lavandier (F.). 1979 b. - Cycle biologique, Régime alimentaire, Production d'Arcynopteryx compacta (Plecoptera, Perlodidae) dans un torrent de haute altitude. Bull. Soc. Hist. Nat. Toulouse, $115: 140-150$.

LAVANDIER (P.). 1981. - Cycle biologique, croissance et production de Rhithrogena loyolaea Navas (Ephemeroptera) dans un torrent pyrénéen de haute mon. tagne. Annls Limnol., 17 (2) : 163-179.

Lavandier (P.) et Mur (C.). 1974. - Ecologie d'un torrent pynénéen de haute montagne II. Caractéristiques chimiques. Annls Limnol., 10 (3) : 275-309.

LILLEHAMER (A.). 1975, - Norwegian stoneflies IV. Laboratory studies on ecological factors influencing distribution. Norw. J. Ent., 22 : 99-108.

Lillehamer. (A.). 1979. - Stonefly populations at high altitudes in Norway. GewässAbwäss., 64: 48-49.

MAdSEN (B. L.), Bengtson (J.) et Butz (I.). 1973. - Observations on upstream migration by imagines of some Plecoptera and Ephemeroptera. Limnol Oceanogr., 18 (4) : 678-681.

Malmoutst (B.) and Sjöström (P.). 1980. - Prey size and feeding patterns in Dinocras cephalotes (Plecoptera) Oikos., 35 (3) : 311-316.

MARKARIAN (R. K.). 1980. - A study of the relationship between aquatic insect growth and water temperature in a small stream. Hydrobiologia, 75 (1) : 8195.

Mouthon (J.) et Verneaux (J.). 1978. - Le rágime alimentaire de Dinocras megacephala (Klapalek) Plecoptera, Perlidae. 14 (3) : 203-214.

Muller. (K.). 1954. - Investigations on the Organic Drift in North swedish streams. Rep. Inst. Freshwat. Res. Drottningholm., 35 : 133-148.

MULLER (K.). 1973. - Life cycles of stream insects. Aquilo, Sen Zool., $14: 105$ 112.

MUlleR (K.). 1982. - The colonization cycle of Freshwater insects. Oecologia, 52 : $202-207$.

Ness (J.) et Dugdale (R. C.). 1959. - Computation of production for populations of aquatic midge larvae. Ecology, $40: 425-430$.

PeCKARSKY (B. L.). 1980. - Predator-prey interactions between stoneflies and mayflies: behavorial observations. Ecology, 61 (4) : 932-943.

Peckarsky (B. L.) et Dodson (S. I.). 1980. - Do stonefly predators influence benthic distributions in streams? Ecology, 61 (6) : 1275-1282.

ScHWARZ (P.). 1970. - Autökologische Untersuchungen Jum Lebenszyklus von setipalpia-Arten (Plecoptera). Arch. Hydrobiol., 67 I : 103-140 et II : 141172.

SHELDON (A. L.). 1969. - Size relationships of Acroneuria californica (Perlidae, plecoptera) and its prey. Hydrobiologia, 34 : 85-94.

SHELDoN (A. L.). 1972. - Comparative ecology of Arcynopteryx and Diura (Plecoptera) in a California stream. Arch. Hydrobiol., 69 : 521-546.

SIEGFRIED (C. A.) and KNIGHT (A. W.). 1976. - Prey selection by a setipalpian stonefly nymph, Acroneuria (Calineuria) californica Banks (Plecoptena : Perlidae). Ecology, 57 : 603-608. 
Sutcliffe (D. W.), Carrick (T. R.) and Willoughby (L. G.). 1981, - Effects of diet, body size, age and temperature on growth rates in the amphipod Gammarus pulex. Freshwater Biology, $11:$ 183-213.

TACHET (H.). 1965. - Recherches sur l'alimentation des larves de Polycentropus (Trichoptères) dans leur milieu naturel. Ann. Soc. Ent. Fr. N. S., 1 (3) : 627633.

WATERS (T.F.). 1969. - The turnover ratio in production ecology of freshwater invertebrates. Amer. Nat., 103 : 173-185.

WATERS (T. F.). 1977. - Secondary production in inland waters. Adv. Ecol. Res., $10: 91-164$.

WATERS (T. F.). 1979. - Influence of benthos life history upon the estimation of secondary production. J. Fish. Res. Board can., 36 (12) : 1425-1430.

WiNBERG (G. G.). 1971. - Methods for the estimation of production of aquatic animals. Academic Press. London.

WINTERBoURN (M. J.). 1974. - The life histories, trophic relations and production of Stenoperla prasina (Plecoptera) and Idelatidium sp. (Ephemeroptera) in a New Zealand river. Freshwater Biology, 4 : 507-524.

Zwick (P.). 1980. - Plecoptera (Steinfliegen) - Handbuch der zoologie IV Band: Arthropoda $-2-$ Hälfte: Insecta (2. Auflage) -2 . Teil : Spezielles, 7 (Lfg. 26) : III + 115 p. Walter de Gruyter, Berlin, New York. 